\title{
Persistence length of a strongly charged rodlike polyelectrolyte in the presence of salt
}

\author{
Gil Ariel and David Andelman* \\ School of Physics and Astronomy, Raymond and Beverly Sackler Faculty of Exact Sciences, Tel-Aviv University, 69978 Tel-Aviv, Israel
}

(Received 18 June 2002; published 30 January 2003)

\begin{abstract}
The persistence length of a single, intrinsically rigid polyelectrolyte chain, above the Manning condensation threshold is investigated theoretically in the presence of added salt. Using a loop expansion method, the partition function is consistently calculated, taking into account corrections to mean-field theory. Within a mean-field approximation, the well-known results of Odijk, Skolnick, and Fixman are reproduced. Beyond mean field, it is found that density correlations between counterions and thermal fluctuations reduce the stiffness of the chain, indicating an effective attraction between monomers for highly charged chains and multivalent counterions. This attraction results in a possible mechanical instability (collapse), alluding to the phenomenon of DNA condensation. In addition, we find that more counterions condense on slightly bent conformations of the chain than predicted by the Manning model for the case of an infinite cylinder. Finally, our results are compared with previous models and experiments.
\end{abstract}

DOI: 10.1103/PhysRevE.67.011805

PACS number(s): 61.25.Hq, 36.20.-r, 87.15.-v

\section{INTRODUCTION}

The behavior of charged polymers has received considerable attention since the early 1970s. However, despite extensive efforts, much of the phenomena observed in systems containing polyelectrolytes (PEs) is still not very well understood [1]. PEs are frequently used in various industrial applications, such as stabilization of charged colloidal suspensions and in flocculation processes. They also are an essential ingredient of many biological systems. These reasons motivated theoretical [2-6], experimental [7-11] and computer simulation [12-15] investigations of PEs. For instance, DNA is known to be a particularly strongly charged polymer, bearing a charge density of one electron charge per $1.7 \AA$.

Despite the strong electrostatic repulsion, PEs show a wide range of complex behavior, depending on the concentration of added salt and its valency. It is observed that with monovalent counterions, PEs are usually stretched and assume a rodlike conformation [16-18]. On the other hand, introduction of a small amount of multivalent counterions significantly reduces the rigidity of the chain [7-10]. Under certain conditions a PE may completely collapse into a globulelike conformation [19-21]. For DNA, this is known as DNA condensation [22].

Even the problem of a single, uniformly charged, polymer in aqueous solution still poses a theoretical challenge [2335]. Single-chain models are much simpler than real experimental systems, as is the case for biopolymers in physiological conditions, or with synthetic PEs. However, much of the interesting phenomena characteristic to dilute PE solutions is still captured in the models despite the extended simplification.

The first breakthrough in treating semiflexible PEs analytically was made by Odijk [30], and independently by Skolnick and Fixman [31] (OSF) by introducing the concept of an "electrostatic persistence length." The notion of persistence length [4], which measures correlations along the

*Electronic address: andelman@post.tau.ac.il chain, is very useful in describing elastic properties of semiflexible polymers in general, and PEs, in particular. According to the OSF theory, the total persistence length of the polymer can be written as a sum of two contributions: the bare persistence length $l_{0}$ and an electrostatic one $l_{e}$. Electrostatic interactions are treated on a mean-field level and the charges on the polymer are assumed to be smeared uniformly. Within a linearized version of the Poisson-Boltzmann theory, the interaction between any two charges is screened and given (in units of $k_{B} T$ ) by the Debye-Hückel expression, $V^{\mathrm{DH}}(\mathbf{r})=l_{B} e^{-\kappa r} / r$. The Bjerrum length $l_{B}=e^{2} / \varepsilon k_{B} T$ is defined as the distance at which the electrostatic interaction between two ions of unit charge $e$ equals the thermal energy $k_{B} T$, where $\varepsilon$ is the dielectric constant of the medium. For water at room temperature, $l_{B} \simeq 7 \AA$. The inverse DebyeHückel screening length is then $\kappa=\left[4 \pi z(z+1) l_{B} c\right]^{1 / 2}$. It depends on the concentration of salt $c$ and the counterion valency $z$, where throughout this paper we will explicitly use a $1: z$ salt. Finally, the polymer is considered to be intrinsically rigid, $l_{0} \gg L$. According to the OSF theory, the electrostatic persistence length then yields

$$
l_{p}=l_{0}+l_{\mathrm{OSF}}=l_{0}+\frac{l_{B} \lambda^{2}}{4 z^{2} \kappa^{2}},
$$

where $\lambda=e / a$ denotes the average line charge density along the chain.

Since the OSF theory is strictly a mean-field theory, the effective interaction between charges on the polymer is always repulsive [32,33]. Indeed, Eq. (1.1) indicates that the polymer becomes more rigid due to electrostatics because $l_{\mathrm{OSF}}>0$. Experiments, however, clearly show that under some conditions, electrostatics may cause a reversed effect $[7-9,22]$, where enhanced chain flexibility results from a negative electrostatic contribution to the persistence length. In order to account for such behavior, corrections to linearized Poisson-Boltzmann have been considered [32-35]. This was done in two steps. The first is to take into account the effect of counterion condensation. With strongly charged 
polymers, some of the counterions are loosely bound to the chain and reduce the effective charge on the polymer. For a straight infinite cylinder, this is known as Manning condensation $[3,36]$. The second step is to consider correlations between the ions and thermal fluctuations of the counterion density [34,35]. Correlations between bound ions become more significant at lower temperatures. In the $T \rightarrow 0$ limit, condensed ions are arranged on a periodic lattice, similar to a Wigner crystal, or a strongly correlated liquid [34]. At higher temperatures such correlations are smeared out due to thermal fluctuations. The latter introduces another correction to mean-field theory coming from induced dipoles, similar to van der Waals interactions [35].

Both correlations and thermal fluctuations are mechanisms that can cause the effective interaction between charges on the polymer to become attractive. Nguyen et al. [34] considered the former and calculated the persistence length of a polymer close to $T=0$. Alternatively, Golestanian et al. [35] considered the fluctuation mechanism. The two models do not agree with each other, and there is still no consensus on the question of which of the mechanisms is more significant at intermediate temperatures, which are used in experiments [37-41].

The aim of the present work is to propose a model which takes into account both correlations and thermal fluctuations of a single, intrinsically rigid, charged polymer, immersed in a bulk and continuous dielectric medium. This will facilitate a closer and consistent examination of the different mechanisms that cause the fundamentally repulsive electrostatic repulsion to become attractive. Since the deviation from meanfield predictions, as seen both in experiments and in previous theoretical works, is so pronounced, an analytical understanding of the problem will be of high value.

In the following section, we introduce our model for treating a single, rodlike $\mathrm{PE}$ in presence of added electrolyte (salt) [42]. In Sec. III, a mean-field approximation is used, and the well-known result of OSF is reproduced. Section IV finds the first-order correction to mean field, taking into account both correlations and thermal fluctuations. This is the main result of the paper where a new expression for the electrostatic persistence length is obtained. This expression accounts for the observed attraction between monomers for strongly charged polymers and multivalent counterions. In the last sections, our results are compared to experiments and other theoretical models. Our findings are further discussed in view of these comparisons.

\section{THE THEORETICAL MODEL}

Consider a polymer chain consisting of $N \gg 1$ monomers of length $a$ each. Taking a worm-like chain approach [4], the polymer is modeled as a spatial curve $\mathbf{R}(s), 0 \leqslant s \leqslant L=N a$, with a total persistence length $l_{p}$. Charges on the chain are assumed to be smeared with a positive constant line charge density $\lambda=e / a$ (one unit charge $e$ per monomer size $a$ ), while mobile ions are taken to be pointlike charges. The system is immersed in a continuous dielectric medium with a dielectric constant $\varepsilon$. For simplicity, we have assumed that coions are monovalent, while counterions are multivalent, carrying a charge $-z e$. Namely, the chain is embedded in a $1: z$ electrolyte solution. In order to account for the effect of counterion (Manning) condensation we follow the two-phase model introduced by Oosawa [3]. The first phase is a 1D gas of counterions that are bound to the polymer and can move only along the chain. The positions of $I$ bound counterions are denoted as $\mathbf{R}\left(s_{1}\right) \cdots \mathbf{R}\left(s_{I}\right)$. The second phase is composed of free counterions in solution, in equilibrium with the 1D gas. Since the Manning-Oosawa model regards the PE chains as an infinite-long cylinder, we will restrict ourselves hereafter to rodlike polymers which satisfy, $l_{p} \gg L$. Finally, we will assume that the effect of the free ions is to screen out all electrostatic interactions $[15,43]$ so that the interaction between any two charges (smeared charges on the polymer and bound $z$-valent counterions) is given by the screened Debye-Hückel interaction: $V^{\mathrm{DH}}(\mathbf{r})=z_{i} z_{j} l_{B} e^{-\kappa r} / r$, where $z_{i}$ and $z_{j}$ are the valencies of the two respective ions. Because we employ a continuum approach, it is necessary to have $\kappa a \ll 1$ ( $a$ is comparable to the size of the smallest molecule in the system-free monovalent ions and monomers). Furthermore, we require that the chain is long enough so that its contour length $L$ is much longer than the screening length, $\kappa L \gg 1$. These limits, $a \ll \kappa^{-1} \ll L$, usually hold in experimental and physiological conditions, and are necessary conditions for our model. In particular, the no added salt limit $(\kappa \rightarrow 0)$ is not covered by our model.

We can proceed by writing down the grand-canonical partition function of the system. Up to a normalization constant it is

$$
\mathcal{Z}=\int \mathcal{D} \mathbf{R}(s) \sum_{I=0}^{\infty} \frac{e^{\mu I}}{I !} \prod_{i=1}^{I}\left(\frac{1}{L} \int_{0}^{L} d s_{i}\right) e^{-H_{0}-H_{\mathrm{int}}}
$$

where the path integral is a sum over all possible spatial conformations of the chain, $\mu$ is the chemical potential of the $1 \mathrm{D}$ gas of bound counterions and is related to the counterion concentration in the bulk reservoir, $H_{0}$ is the Hamiltonian of a neutral chain with bare persistence length $l_{0}$, and $H_{\text {int }}$ is the electrostatic interaction Hamiltonian. It consists of screened electrostatic interactions between all charged monomers and bound counterions and is written as a sum of three different contributions: $H_{i n t}=H_{m m}+H_{b b}+H_{m b}$, where

$$
\begin{gathered}
H_{m m}=\frac{1}{2} \frac{1}{a^{2}} \int_{0}^{L} \int_{0}^{L} d s d s^{\prime} V^{\mathrm{DH}}\left(\mathbf{R}(s)-\mathbf{R}\left(s^{\prime}\right)\right), \\
H_{m b}=\frac{1}{a} \int_{0}^{L} d s \sum_{i=1}^{I} V^{\mathrm{DH}}\left(\mathbf{R}(s)-\mathbf{R}\left(s_{i}\right)\right), \\
H_{b b}=\frac{1}{2} \sum_{i=1}^{I} \sum_{j=1}^{I} V^{\mathrm{DH}}\left(\mathbf{R}\left(s_{i}\right)-\mathbf{R}\left(s_{j}\right)\right) .
\end{gathered}
$$

All energies are dimensionless and written in terms of the thermal energy $k_{B} T$. In this form, the integrations of Eq. (2.2) diverge as the terms contain also self-interactions (for instance, when $s \rightarrow s^{\prime}$ in $H_{m m}$ ). All integrations, therefore, should have a lower cutoff at a distance of order $a$. 
In order to treat the interaction term analytically, it is more convenient to use continuous volume concentrations defined in the following way [44-47]

$$
\begin{gathered}
\phi^{m}(\mathbf{r})=\frac{1}{a} \int_{0}^{L} d s \delta(\mathbf{r}-\mathbf{R}(s)), \\
\phi^{b}(\mathbf{r})=\sum_{i=1}^{I} \delta\left(\mathbf{r}-\mathbf{R}\left(s_{i}\right)\right),
\end{gathered}
$$

where $\phi^{m}$ and $\phi^{b}$ are the monomer and bound counterion concentrations at location $\mathbf{r}$, respectively. These can be substituted into the partition function, Eq. (2.1), by making use of the identity operator that couples discrete and continuous concentrations. This is done using the path integral representation of the Dirac delta function

$$
\begin{aligned}
1= & \int \mathcal{D} \phi^{m}(\mathbf{r}) \delta\left[\phi^{m}(\mathbf{r})-\frac{1}{a} \int_{0}^{L} d s \delta(\mathbf{r}-\mathbf{R}(s))\right] \\
= & \int \mathcal{D} \phi^{m}(\mathbf{r}) \mathcal{D} \psi^{m}(\mathbf{r}) \exp \left\{-i \int d^{3} \mathbf{r} \psi^{m}(\mathbf{r})\right. \\
& \left.\times\left[\phi^{m}(\mathbf{r})-\frac{1}{a} \int_{0}^{L} d s \delta(\mathbf{r}-\mathbf{R}(s))\right]\right\} \\
1= & \int \mathcal{D} \phi^{b}(\mathbf{r}) \delta\left[\phi^{b}(\mathbf{r})-\sum_{k=1}^{I} \delta\left(\mathbf{r}-\mathbf{R}\left(s_{k}\right)\right)\right] \\
= & \int \mathcal{D} \psi^{b}(\mathbf{r}) \mathcal{D} \phi^{b}(\mathbf{r}) \exp \left\{-i \int d^{3} \mathbf{r} \psi^{b}(\mathbf{r})\right. \\
& \left.\times\left[\phi^{b}(\mathbf{r})-\sum_{k=1}^{I} \delta\left(\mathbf{r}-\mathbf{R}\left(s_{k}\right)\right)\right]\right\} .
\end{aligned}
$$

The extra complexity of this method is the introduction of two new auxiliary fields, denoted $\psi^{m}$ and $\psi^{b}$, which couple to $\phi^{m}$ and $\phi^{b}$, respectively. Substituting Eqs. (2.4) and (2.3) into Eq. (2.1), the partition function reads

$$
\begin{aligned}
& \mathcal{Z}=\int \mathcal{D} \mathbf{R}(s)\left(\prod_{i=m, b} \mathcal{D} \phi^{i} \mathcal{D} \psi^{i} \xi_{i}[\mathbf{R}]\right) \exp \left(-H_{\text {cont }}\right), \\
& \xi_{m}[\mathbf{R}]=\exp \left[-H_{i d}+\frac{i}{a} \int_{0}^{L} d s \psi^{m}(\mathbf{R}(s))\right. \\
& \left.+i \int d^{3} \mathbf{r} \phi^{m}(\mathbf{r}) \psi^{m}(\mathbf{r})\right] \\
& \xi_{b}[\mathbf{R}]=\exp \left\{\int d^{3} \mathbf{r}\left[a n_{0} e^{i \psi^{b}(\mathbf{r})} \phi^{m}(\mathbf{r})+i \phi^{b}(\mathbf{r}) \psi^{b}(\mathbf{r})\right]\right\}, \\
& H_{\mathrm{cont}}=\frac{1}{2} \iint d^{3} \mathbf{r} d^{3} \mathbf{r}^{\prime} \boldsymbol{\Phi}(\mathbf{r}) \hat{Z} \boldsymbol{\Phi}\left(\mathbf{r}^{\prime}\right) V^{\mathrm{DH}}\left(\mathbf{r}-\mathbf{r}^{\prime}\right), \\
& \boldsymbol{\Phi}=\left(\begin{array}{c}
\phi^{m} \\
\phi^{b}
\end{array}\right), \quad \hat{Z}=\left(\begin{array}{cc}
1 & -z \\
-z & z^{2}
\end{array}\right) \text {. }
\end{aligned}
$$

where we have defined

$$
n_{0}=e^{\mu} / L
$$

and $\xi_{b}$ was simplified in the following way:

$$
\begin{aligned}
\xi_{b}= & \sum_{I=0}^{\infty} \frac{1}{I !} e^{\mu I} \prod_{i=1}^{I}\left(\int_{0}^{L} \frac{d s_{i}}{L}\right) \\
& \times \exp \left[i \int^{3} \mathbf{r} \psi^{b}(\mathbf{r})\left(\sum_{k=1}^{I} \delta\left(\mathbf{r}-\mathbf{R}\left(s_{k}\right)\right)\right)\right] \\
= & \sum_{I=0}^{\infty} \frac{1}{I !} e^{\mu I} \prod_{i=1}^{I}\left(\int_{0}^{L} \frac{d s_{i}}{L}\right) \exp \left[i \sum_{k=1}^{I} \psi^{b}\left(\mathbf{R}\left(s_{k}\right)\right)\right] \\
= & \exp \left[n_{0} \int_{0}^{L} d s e^{i \psi^{b}(\mathbf{R}(s))}\right] \\
= & \exp \left[a n_{0} \int d^{3} \mathbf{r} e^{i \psi^{b}(\mathbf{r})} \phi^{m}(\mathbf{r})\right] .
\end{aligned}
$$

It is easily seen that carrying out the integrations over the new fields $\phi^{m}, \psi^{m}, \phi^{b}, \psi^{b}$ reproduces Eq. (2.1) exactly. However, the form of the continuous partition function, Eq. (2.5), is better organized: single-body interactions of the monomer concentration $\phi^{m}$ and the bound counterion concentration $\phi^{b}$ are collected into the terms $\xi_{m}$ and $\xi_{b}$. The two-body interaction term $H_{\text {cont }}$ has the form of a quadratic interaction between the concentrations vector field $\boldsymbol{\Phi}$, where the interaction between the two vector fields $\boldsymbol{\Phi}(\mathbf{r})$ and $\boldsymbol{\Phi}\left(\mathbf{r}^{\prime}\right)$ is given by the matrix $\hat{Z} V^{\mathrm{DH}}\left(\mathbf{r}-\mathbf{r}^{\prime}\right)$. In the above equation, we use vector notation to write the interaction between the different fields as

$$
\begin{aligned}
\boldsymbol{\Phi}(\mathbf{r}) \hat{Z} \boldsymbol{\Phi}\left(\mathbf{r}^{\prime}\right) V^{\mathrm{DH}}\left(\mathbf{r}-\mathbf{r}^{\prime}\right)= & {\left[\boldsymbol{\phi}^{m}(\mathbf{r}) \phi^{m}\left(\mathbf{r}^{\prime}\right)-2 z \phi^{m}(\mathbf{r}) \phi^{b}\left(\mathbf{r}^{\prime}\right)\right.} \\
& \left.+z^{2} \phi^{b}(\mathbf{r}) \phi^{b}\left(\mathbf{r}^{\prime}\right)\right] V^{\mathrm{DH}}\left(\mathbf{r}-\mathbf{r}^{\prime}\right) .
\end{aligned}
$$

This method can be easily generalized in order to account for any additional species the system may contain, or to different types of (nonelectrostatic) interactions. For instance, a local interaction can be added to the matrix elements of $\hat{Z} V^{\mathrm{DH}}\left(\mathbf{r}-\mathbf{r}^{\prime}\right)$ in order to include excluded volume interactions. If counterions are replaced by more complex charged amphiphiles, a hydrophobic attraction between the species can be added in a similar manner:

$$
\left(\begin{array}{cc}
1 & -z \\
-z & z^{2}
\end{array}\right) V^{\mathrm{DH}}\left(\mathbf{r}-\mathbf{r}^{\prime}\right)+\left(\begin{array}{cc}
v_{m m} & v_{m b} \\
v_{m b} & v_{b b}
\end{array}\right) \delta\left(\mathbf{r}-\mathbf{r}^{\prime}\right),
$$

where $v_{i j}(i, j=m$ or $b)$ denote second viral coefficients.

So far, the partition function Eq. (2.5) is exact up to the general assumptions of the model-wormlike polymer, smeared charges on the chain, separation into two phases and screening by free ions. However, the integrations cannot be carried out analytically and some approximations have to be made. The first-order correction just reproduces the wellknown mean-field results, as will be shown below in Sec. III. 
Higher-order terms in the expansion represent corrections to mean field and will be presented in Sec. IV.

\section{THE MEAN-FIELD APPROXIMATION}

The approximation method we use is a systematic expansion in powers of the auxiliary field $\psi^{b}$, similar to loop expansion in field theory $[44,45]$. Expanding to first order in $\psi^{b}$ results in a mean-field approximation. The partition function takes into account the average interaction between the monomers and the bound counterions [44]. Note that this method is somewhat different than calculating the zeroth-order saddle-point approximation of the integral over $\mathcal{D} \mathbf{R}(s)$ in Eq. (2.5). For a detailed comparison between the two methods see Ref. [45].

Expanding to first order in $\psi^{b}$, appearing only in $\xi_{b}$ we obtain

$$
\xi_{b} \simeq \exp \left\{\int d^{3} \mathbf{r}\left[a n_{0}\left(1+i \psi^{b}(\mathbf{r})\right) \phi^{m}(\mathbf{r})+i \phi^{b}(\mathbf{r}) \psi^{b}(\mathbf{r})\right]\right\} .
$$

Applying a Fourier transform the partition function reads

$$
\begin{gathered}
\mathcal{Z}_{1}=\int \mathcal{D} \mathbf{R}(s)\left(\prod_{i=m, b} \mathcal{D} \phi^{i} \mathcal{D} \psi^{i}\right) \xi_{m} e^{-H_{1}} \\
H_{1}=\int \frac{d^{3} \mathbf{k}}{(2 \pi)^{3}}\left[\frac{1}{2} \boldsymbol{\Phi}_{\mathbf{k}}^{\dagger} \hat{Z} \boldsymbol{\Phi}_{-\mathbf{k}} V_{\mathbf{k}}^{\mathrm{DH}}+i \phi_{\mathbf{k}}^{b} \psi_{-\mathbf{k}}^{b}-i a n_{0} \phi_{\mathbf{k}}^{m} \psi_{-\mathbf{k}}^{b}\right. \\
\left.-a n_{0} \phi_{\mathbf{k}}^{m}(2 \pi)^{3} \delta(\mathbf{k})\right]
\end{gathered}
$$

where $\mathcal{Z}_{1}$ is the partition function up to first order in $\psi^{b}$. The Fourier transform of $\phi^{b}(\mathbf{r}), \psi^{b}(\mathbf{r})$ and $\phi^{m}(\mathbf{r})$, is denoted by $\phi_{\mathbf{k}}^{b}, \psi_{\mathbf{k}}^{b}$ and $\phi_{\mathbf{k}}^{m}$, respectively. The interaction Hamiltonian $H_{1}$ consists of three contributions: the first term is the twobody Debye-Hückel interactions. The second term is a bilinear coupling of the concentration fields, $\phi^{m}$ and $\phi^{b}$, with the auxiliary field $\psi^{b}$, generated by the bound counterions. As for the third term, we will later show that it is only a constant. The integrations over the degrees of freedom of the bound ions $\left\{\phi^{b}, \psi^{b}\right\}$ can now be carried out:

$$
\begin{aligned}
\mathcal{Z}_{1}= & \int \mathcal{D} \mathbf{R}(s) \mathcal{D} \phi^{m} \mathcal{D} \psi^{m} \xi_{m} e^{-H_{\mathrm{eff}, 1}} \\
H_{\mathrm{eff}, 1}= & \int \frac{d^{3} \mathbf{k}}{(2 \pi)^{3}}\left[\frac{1}{2}\left(1-a n_{0} z\right)^{2} V_{\mathbf{k}}^{\mathrm{DH}} \phi_{\mathbf{k}}^{m} \phi_{-\mathbf{k}}^{m}\right. \\
& \left.-a n_{0} \phi_{\mathbf{k}}^{m}(2 \pi)^{3} \delta(\mathbf{k})\right] .
\end{aligned}
$$

The effective interaction Hamiltonian between the monomers, $H_{\text {eff }, 1}$, is correct up to first order in $\psi^{b}$. The first term of $H_{\text {eff, } 1}$ consists of screened electrostatic interaction $\left(V^{\mathrm{DH}}\right)$ between Fourier components of the monomer concentration field $\phi^{m}$. The interaction includes a reduced charge density of $\left(1-a n_{0} z\right)$. Note that $H_{\text {eff, } 1}$ depends on the conformation of the polymer $\mathbf{R}(s)$ through the definition of $\phi^{m}$, Eq. (2.3).

\section{A. Averages and correlations}

After presenting the partition function, our aim is to integrate out the degrees of freedom of bound ions and obtain averaged quantities up to first order in $\psi^{b}$. Averages over configurations of the 1D gas of bound ions are defined as

$$
\begin{gathered}
\mathcal{Z}_{1}=\operatorname{Tr}_{\left\{\mathbf{R}, \phi^{m}, \psi^{m}\right\}} \xi_{m} \operatorname{Tr}_{\left\{\phi^{b}, \psi^{b}\right\}} e^{-H_{1}}=\operatorname{Tr}_{\left\{\mathbf{R}, \phi^{m}, \psi^{m}\right\}} \xi_{m} e^{-H_{\text {eff }, 1}} \\
\langle\mathcal{O}\rangle_{1}=\frac{\operatorname{Tr}_{\left\{\phi^{b}, \psi^{b}\right\}} \mathcal{O} e^{-H_{1}}}{\operatorname{Tr}_{\left\{\phi^{b}, \psi^{b}\right\}} e^{-H_{1}}}=\frac{\operatorname{Tr}_{\left\{\phi^{b}, \psi^{b}\right\}} \mathcal{O} e^{-H_{1}}}{e^{-H_{\text {eff }, 1}}}
\end{gathered}
$$

Taking derivatives of the partition function, different averages and correlation functions can be calculated. For instance

$$
\begin{gathered}
\left\langle\psi_{\mathbf{k}}^{b}\right\rangle_{1}=i z\left(1-a n_{0} z\right) V_{\mathbf{k}}^{\mathrm{DH}} \phi_{\mathbf{k}}^{m}, \\
\left\langle\psi_{\mathbf{k}_{1}}^{b} \psi_{\mathbf{k}_{2}}^{b}\right\rangle_{1}=\left\langle\psi_{\mathbf{k}_{1}}^{b}\right\rangle_{1}\left\langle\psi_{\mathbf{k}_{2}}^{b}\right\rangle_{1}-(2 \pi)^{3} z^{2} V_{\mathbf{k}_{1}}^{\mathrm{DH}} \delta\left(\mathbf{k}_{1}+\mathbf{k}_{2}\right), \\
\left\langle\phi_{\mathbf{k}_{1}}^{b} \phi_{\mathbf{k}_{2}}^{b}\right\rangle_{1}=\left\langle\phi_{\mathbf{k}_{1}}^{b}\right\rangle_{1}\left\langle\phi_{\mathbf{k}_{2}}^{b}\right\rangle_{1}, \\
\left\langle\phi_{\mathbf{k}_{1}}^{b} \psi_{\mathbf{k}_{2}}^{b}\right\rangle_{1}=\left\langle\phi_{\mathbf{k}_{1}}^{b}\right\rangle_{1}\left\langle\psi_{\mathbf{k}_{2}}^{b}\right\rangle_{1}-i(2 \pi)^{3} \delta\left(\mathbf{k}_{1}+\mathbf{k}_{2}\right) .
\end{gathered}
$$

It is interesting to observe that the effective interaction Hamiltonian $H_{\text {eff, } 1}$ of Eq. (3.3) can be rewritten as

$$
\begin{aligned}
H_{\mathrm{eff}, 1}= & \int \frac{d^{3} \mathbf{k}}{(2 \pi)^{3}}\left[-\frac{i}{2 z}\left(1-a n_{0} z\right)\left\langle\psi_{\mathbf{k}}^{b}\right\rangle_{1} \phi_{-\mathbf{k}}^{m}\right. \\
& \left.-a n_{0} \phi_{\mathbf{k}}^{m}(2 \pi)^{2} \delta(\mathbf{k})\right] .
\end{aligned}
$$

Up to a constant prefactor, the first term of $H_{\text {eff, } 1}$ is the interaction of the monomer concentration field $\phi^{m}$ with the auxiliary field $\psi^{b}$ averaged within mean-field approximation. Therefore, it is the averaged interaction between each monomer and the auxiliary field produced by the counterions.

\section{B. Density of bound ions}

In the Manning-Oosawa model [3,36], the PE is considered an infinite charged cylinder, and the average number of bound counterions of valency $z$ per unit length is

$$
n^{M}=\frac{1}{z}\left[\frac{1}{a}-\frac{1}{z l_{B}}\right]=\frac{q-1}{z^{2} l_{B}},
$$

where the dimensionless parameter $q=z l_{B} / a$ is used in Eq. (3.7). Condensation of $z$-valent counterions occurs for $q>1$ and effectively lowers the value of $q$ to unity, $q_{\text {eff }}=1$ [48]. Note that below the Manning condensation threshold ( $q$ $<1$ ) one should simply set $n^{M}=0$.

The average number of bound counterions per unit length 


$$
n^{\mathrm{tot}}[\mathbf{R}]=\frac{1}{L} \int d^{3} \mathbf{r} \phi^{b}(\mathbf{r}) .
$$

where the dependence on the polymer conformation, $\mathbf{R}(s)$, is through the definition of $\phi^{b}$, Eq. (2.3). Since we treat here only single polymer chains, the system is assumed to be infinitely dilute, in the sense that each PE chain occupies only a small fraction of the overall system volume. Small changes in the polymer conformation are not expected to change the chemical potential of the free counterion gas which occupies the entire volume. As the 1D phase of bound counterions is in equilibrium with the free counterion phase, their chemical potential is equal. We conclude that $\mu$, and consequently $n_{0}=\mathrm{e}^{\mu} / L$, should not depend on the conformation of the chain in the dilute polymer limit. Of particular interest is the straight-rod conformation. In this conformation, the density of bound counterions should be consistent with the Manning theory, and $n^{\text {tot }}[\mathbf{R}]$ should, therefore, satisfy

$$
n^{\text {tot }}[\operatorname{rod}]=n^{M}
$$

where $n^{M}$ is the Manning value given in Eq. (3.7). Within the mean-field approximation, the average number of bound counterions per unit length $n_{1}^{\text {tot }}$ can be obtained by substituting Eq. (2.3) and Eq. (3.5) into Eq. (3.8)

$$
\begin{aligned}
n_{1}^{\mathrm{tot}}[\mathbf{R}] & =\frac{1}{L} \int d^{3} \mathbf{r}\left\langle\phi^{b}(\mathbf{r})\right\rangle_{1}=\frac{1}{L} \int d^{3} \mathbf{r} \int \frac{d^{3} \mathbf{k}}{(2 \pi)^{3}} e^{i \mathbf{k} \cdot \mathbf{r}} a n_{0} \phi_{\mathbf{k}}^{m} \\
& =\frac{a n_{0}}{L} \int d^{3} \mathbf{r} \int \frac{d^{3} \mathbf{k}}{(2 \pi)^{3}} e^{i \mathbf{k} \cdot \mathbf{r}} \frac{1}{a} \int_{0}^{L} d s e^{i \mathbf{k} \cdot \mathbf{R}(s)} \\
& =\frac{n_{0}}{L} \int d^{3} \mathbf{k} \int_{0}^{L} d s e^{i \mathbf{k} \cdot \mathbf{R}(s)} \delta(\mathbf{k})=\frac{1}{L} n_{0} \int_{0}^{L} d s=n_{0} .
\end{aligned}
$$

Equation (3.10) gives us the connection between the average density of the bound counterions, $n^{\text {tot }}[\mathbf{R}]$, and the chemical potential, $\mu$ (through $n_{0}$ ), up to first order in $\psi^{b}$. According to $(3.10), n_{1}^{\text {tot }}[\mathbf{R}]$ does not depend on the conformation of the polymer and is just equal to the concentration $n_{0}$. In the following section we will see that this is strictly a meanfield result. Comparing Eqs. (3.9) and (3.10) we find that $n_{0}=n^{M}$.

\section{The persistence length}

Substituting $n_{0}$ into the effective interaction, Eq. (3.3), yields

$$
\begin{aligned}
H_{\mathrm{eff}, 1} & =\frac{1}{2}\left(1-a z n^{M}\right)^{2} \int \frac{d^{3} \mathbf{k}}{(2 \pi)^{3}} V_{\mathbf{k}}^{\mathrm{DH}} \boldsymbol{\phi}_{\mathbf{k}}^{m} \boldsymbol{\phi}_{-\mathbf{k}}^{m}-L n^{M} \\
& =\frac{L}{2 z^{2} l_{B}} \chi[\mathbf{R}]-L n^{M}
\end{aligned}
$$

where

$$
\chi[\mathbf{R}]=\frac{1}{L} \int_{0}^{L} d s \int_{0}^{L} d s^{\prime} \frac{e^{-\kappa\left|\mathbf{R}(s)-\mathbf{R}\left(s^{\prime}\right)\right|}}{\left|\mathbf{R}(s)-\mathbf{R}\left(s^{\prime}\right)\right|} .
$$

Up to a constant, the integrand is just the screened Coulomb interaction between any two monomers. The integral is a sum over all such monomer pairs along the chain with charge density set at the Manning value $(q=1$ is equivalent to $\lambda$ $\left.=e / a=e / z l_{B}\right)$. This is exactly the interaction Hamiltonian assumed by OSF for a polymer carrying a uniform line charge density of $\lambda=e / z l_{B}$.

Odijk's method for calculating the electrostatic persistence length [30] assumes small, constant curvature deformations from the straight rod conformation. The persistence length is then obtained from the rigidity coefficient of a semiflexible rod. The procedure will be described in greater detail in the following section. Using the effective Hamiltonian $H_{\text {eff,1 }}$ of Eq. (3.11), the OSF result, Eq. (1.1) is reproduced, with the average density of bound counterions as predicted by Manning:

$$
\begin{gathered}
l_{p}=l_{0}+l_{e, 1}, \\
l_{e, 1}=l_{\mathrm{OSF}}=\frac{1}{4 z^{2} \kappa^{2} l_{B}} .
\end{gathered}
$$

\section{BEYOND MEAN FIELD}

The results obtained in the preceding section are strictly on a mean-field level $[44,45]$. In order to go beyond this approximation, higher than linear powers of $\psi^{b}$ have to be included in the partition function, Eq. (2.1). The exact partition function, Eq. (2.1), can be rewritten as

$$
\begin{gathered}
\mathcal{Z}=\operatorname{Tr}_{\left\{\mathbf{R}, \phi^{m}, \psi^{m}\right\}} \xi_{m} e^{-H_{\mathrm{eff}, 1}\left\langle e^{-\Delta H}\right\rangle_{1},} \\
\Delta H=-\operatorname{an}_{0} \int d^{3} \mathbf{r} \phi^{m}(\mathbf{r}) \\
\times\left[\frac{i^{2}}{2 !}\left(\psi^{b}(\mathbf{r})\right)^{2}+\cdots+\frac{i^{n}}{n !}\left(\psi^{b}(\mathbf{r})\right)^{n}+\cdots\right] .
\end{gathered}
$$

Performing a cumulant expansion

$$
\begin{aligned}
\mathcal{Z}= & \operatorname{Tr}_{\left\{\mathbf{R}, \phi^{m}, \psi^{m}\right\}} \xi_{m} e^{-H_{\text {eff }, 1} \exp }\left[-\langle\Delta H\rangle_{c, 1}+\frac{1}{2 !}\left\langle\Delta H^{2}\right\rangle_{c, 1}\right. \\
& \left.-\frac{1}{3 !}\left\langle\Delta H^{3}\right\rangle_{c, 1}+\cdots\right]
\end{aligned}
$$

where $\left\langle\mathcal{O}^{n}\right\rangle_{\mathrm{c}, 1}$ denotes the $n$th cumulant. The subscript 1 indicates that the cumulants are calculated using the firstorder expansion of $\Delta H$. For instance, $\left\langle\mathcal{O}^{2}\right\rangle_{\mathrm{c}, 1}=\left(\left\langle\mathcal{O}^{2}\right\rangle_{1}\right.$ $\left.-\langle\mathcal{O}\rangle_{1}^{2}\right)$, where the moments $\left\langle\mathcal{O}^{n}\right\rangle_{1}$ are defined according to Eq. (3.4).

The effective interaction not included in $H_{\mathrm{eff}, 1}$ of Eq. (3.11) is therefore 


$$
\langle\Delta H\rangle_{c, 1}-\frac{1}{2 !}\left\langle\Delta H^{2}\right\rangle_{c, 1}+\frac{1}{3 !}\left\langle\Delta H^{3}\right\rangle_{c, 1}+\cdots
$$

\section{A. Second-order corrections}

The only term in Eq. (4.3) which is of second order in $\psi^{b}$ is the first term of the first cumulant:

$$
H_{2}=\frac{1}{2} a n_{0} \int d^{3} \mathbf{r} \phi^{m}(\mathbf{r})\left\langle\left[\psi^{b}(\mathbf{r})\right]^{2}\right\rangle_{1} .
$$

Applying a Fourier transform, $\mathrm{H}_{2}$ can be expressed in Fourier space:

$$
H_{2}=\frac{1}{2} a n_{0} \int \frac{d^{3} \mathbf{k}_{1}}{(2 \pi)^{3}} \frac{d^{3} \mathbf{k}_{2}}{(2 \pi)^{3}}\left\langle\psi_{\mathbf{k}_{1}}^{b} \psi_{\mathbf{k}_{2}}^{b}\right\rangle_{1} \phi_{-\mathbf{k}_{1}-\mathbf{k}_{2}}^{m} .
$$

Substituting $\phi_{\mathbf{k}}^{m}$ and $\psi_{\mathbf{k}}^{b}$ into the correlation expression, Eq. (3.5), yields a correction to the effective interaction Hamiltonian $H_{\text {eff }, 1}$ obtained in Eq. (3.11)

$$
\begin{aligned}
H_{\mathrm{eff}, 2}= & -\frac{1}{2} z^{2} \frac{n_{0} l_{B}^{2}}{a^{2}}\left(1-a z n_{0}\right)^{2}\left[\int_{0}^{L} d s_{0} \int_{0}^{L} d s_{1} \int_{0}^{L} d s_{2}\right. \\
& \left.\times \frac{e^{-\kappa\left|\mathbf{R}\left(s_{1}\right)-\mathbf{R}\left(s_{0}\right)\right|}}{\left|\mathbf{R}\left(s_{1}\right)-\mathbf{R}\left(s_{0}\right)\right|} \frac{e^{-\kappa\left|\mathbf{R}\left(s_{2}\right)-\mathbf{R}\left(s_{0}\right)\right|}}{\left|\mathbf{R}\left(s_{2}\right)-\mathbf{R}\left(s_{0}\right)\right|}\right]+z^{2} l_{B} n_{0} N .
\end{aligned}
$$

The above result is obtained by using a lower cutoff at distance $a$ on one of the three integrations, and expanding the integral in powers of $\kappa a$, neglecting all but the leading term. In the expansion method, $\psi^{b}$ represents an auxiliary field that is generated by the bound counterions $[27,44]$. Examining the mean-field interaction, Eq. (4.5), each mode of the monomer concentration field $\phi^{m}$ interacts with the average of two $\psi^{b}$ auxiliary fields. The result is a counterion-monomercounterion interaction, and appropriately, the first term of $H_{\text {eff,2 }}$ in Eq. (4.6) has the form of a three-body interaction. As explained in the previous section, the chemical potential $\mu$ does not depend on the polymer conformation $\mathbf{R}(s)$. As a consequence, the second term of $H_{\text {eff, } 2}$ in Eq. (4.6) does not depend on the conformation $\mathbf{R}(s)$ and does not contribute to the persistence length.

\section{B. Density of bound ions}

Taking into account second-order corrections, the average number of bound counterions changes as well

$$
n_{2}^{\mathrm{tot}}[\mathbf{R}]=\frac{1}{L} \int d^{3} \mathbf{r}\left\langle\phi^{b}(\mathbf{r})\right\rangle_{2}=n_{0}+n_{0} z\left(1-a z n_{0}\right) \frac{l_{B}}{a} \chi[\mathbf{R}],
$$

where $\langle\mathcal{O}\rangle_{2}$ denotes the average of $\mathcal{O}$ calculated with the second-order Hamiltonian, $\mathrm{H}_{2}$ of Eq. (4.5).
According to the theory of Manning condensation, the density of bound counterions for a straight rod is $n_{2}^{\text {tot }}[\mathrm{rod}]$ $=n^{M}[48,49]$. The chemical potential $\mu$, defined through $n_{0}$, should therefore satisfy

$$
a n_{0}+n_{0} z\left(1-a z n_{0}\right) l_{B} \chi[\mathrm{rod}]=a n^{M},
$$

where $\chi[\operatorname{rod}]$ is the value of $\chi[\mathbf{R}]$ for the straight rod conformation, in which $\left|\mathbf{R}(s)-\mathbf{R}\left(s^{\prime}\right)\right|=\left|s-s^{\prime}\right|$. Substituting in Eq. (4.8) the expression for $n^{M}$, Eq. (3.7), yields

$$
n_{0}=\frac{q \chi[\mathrm{rod}]+1 \pm \sqrt{(q \chi[\mathrm{rod}]-1)^{2}+4 \chi[\mathrm{rod}]}}{2 z^{2} l_{B} \chi[\mathrm{rod}]} .
$$

In the limit of extremely high salt concentrations, $\kappa a \gg 1$, all correlation and fluctuation effects are screened out completely and decay exponentially with $\kappa a$. The smaller of the two solutions of Eq. (4.8) is therefore chosen in order that the Manning value $n_{2}^{\text {tot }}[\mathbf{R}]=n^{M}$ is reproduced: $n_{0}[\kappa a \rightarrow \infty]$ $=n^{M}$. As explained in the preceding section, the chemical potential $\mu$ (and consequently $n_{0}=e^{\mu} / L$ ) is not expected to depend on the polymer conformation. The density of the bound counterions $n_{2}^{\text {tot }}[\mathbf{R}]$ does, however, depend on the polymer conformation through Eq. (4.7).

\section{Persistence length}

Using the effective Hamiltonian, Eq. (4.6), and the chemical potential, Eq. (4.9), it is now possible to repeat Odijk's method and calculate the electrostatic persistence length. The difference between the effective interaction energy at a general conformation $\mathbf{R}(s)$ as compared to the straight-rod one is

$$
\Delta H_{\mathrm{eff}}=H_{\mathrm{eff}, 1}[\mathbf{R}]+H_{\mathrm{eff}, 2}[\mathbf{R}]-H_{\mathrm{eff}, 1}[\operatorname{rod}]-H_{\mathrm{eff}, 2}[\operatorname{rod}] .
$$

Odijk's method for calculating the persistence length requires expanding $\Delta H_{\text {eff }}$ in small deformations of the chain around the straight-rod conformation [30]. In this limit, the distance between points $s$ and $s^{\prime}$ can be approximated as

$$
\begin{gathered}
\left|\mathbf{R}(s)-\mathbf{R}\left(s^{\prime}\right)\right| \simeq\left|s-s^{\prime}\right|\left[1-\alpha\left(s, s^{\prime}\right)\right]+O\left(\alpha^{2}\right), \\
\alpha\left(s, s^{\prime}\right)=\frac{1}{24}\left(\frac{s-s^{\prime}}{\rho}\right)^{2}
\end{gathered}
$$

where $\rho \gg L$ is the small overall radius of curvature of the fluctuating chain (not to be confused with a spontaneous radius of curvature). The persistence length is then given by

$$
l_{e, 2}=2 \frac{\rho^{2}}{L} \Delta H_{\mathrm{eff}} \cdot
$$

It is, therefore, additive in terms of $\Delta H_{\text {eff }}$ : 


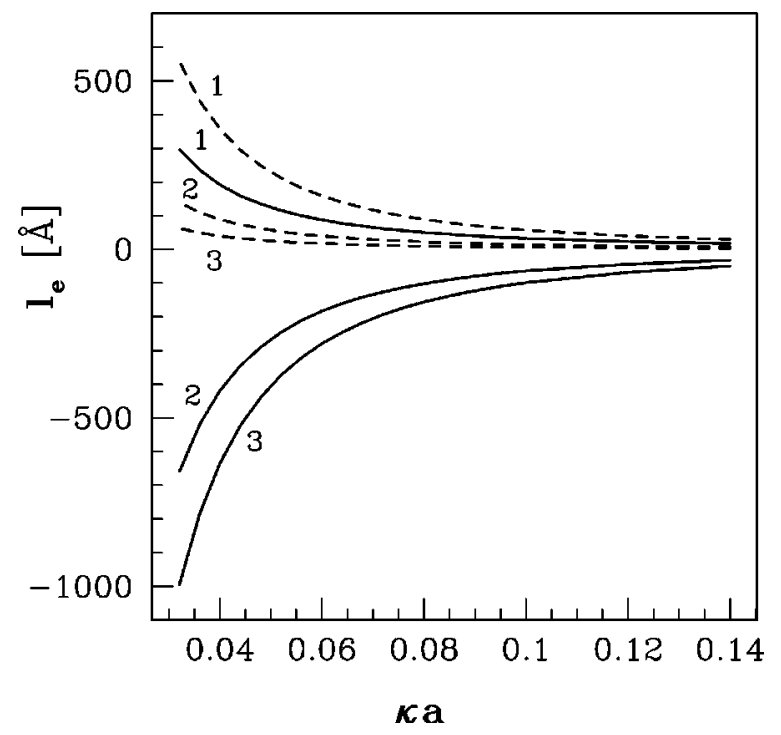

FIG. 1. The electrostatic persistence length $l_{e}$ as function of $\kappa a$ according to OSF (dashed line) and our $l_{e, 2}$ of Eq. (4.14) (solid line). Valencies are specified next to each curve. The parameters chosen are: $a=4 \AA, l_{B}=7 \AA$, so that $q=1.75 z$. The negative $l_{e}$ values for $z=2,3$ indicate a possible collapse transition of the PE chain.

$$
\begin{aligned}
l_{e, 2}= & 2 \frac{\rho^{2}}{L}\left(H_{\mathrm{eff}, 1}[\mathbf{R}]-H_{\mathrm{eff}, 1}[\operatorname{rod}]\right) \\
& +2 \frac{\rho^{2}}{L}\left(H_{\mathrm{eff}, 2}[\mathbf{R}]-H_{\mathrm{eff}, 2}[\operatorname{rod}]\right)
\end{aligned}
$$

On the other hand, we note that the electrostatic persistence length is not additive in orders of $\psi^{b}\left[l_{e, 1} \neq\right.$ $\left.2 \rho^{2} / L\left(H_{\mathrm{eff}, 1}[\mathbf{R}]-H_{\mathrm{eff}, 1}[\mathrm{rod}]\right)\right]$ as the expression for the chemical potential also changes, as compared to the meanfield approximation. Inserting in Eq. (4.13) the value for $n_{0}$, Eq. (4.9), and expanding the result in powers of $1 / \rho$ the different integrations can be evaluated. This requires cutting of all ultraviolet divergences at a distance $a$. In the limit of $a \ll \kappa^{-1} \ll L$ we find

$$
\begin{gathered}
l_{p}=l_{0}+l_{e, 2}, \\
l_{e, 2}=l_{\mathrm{OSF}}\left[q(2-q)-\frac{(q-1)^{2}}{q \ln \kappa a}\right],
\end{gathered}
$$

where we have expanded $l_{e, 2}$ in $(\ln \kappa a)^{-1}$ and kept the two leading terms. Note that we have already taken the first-order (mean-field) interaction into account in Eq. (4.10). Equation (4.14) is our main prediction and is depicted in Fig. 1 for different counterion valencies $z=1,2,3$ as a function of $\kappa a$. At low salt concentrations ( $\kappa a \ll 1$ ) or high $q$, the persistence length maintains the OSF $\kappa^{-2}$ dependence, $l_{e} \sim l_{\mathrm{OSF}} \sim \kappa^{-2}$. We find that the electrostatic persistence length depends strongly on the valency of the counterions. For monovalent counterions $l_{e}$ is usually positive, indicating an effective repulsion between the monomers. However, its value is smaller than that predicted by OSF. Introduction of multivalent coun-

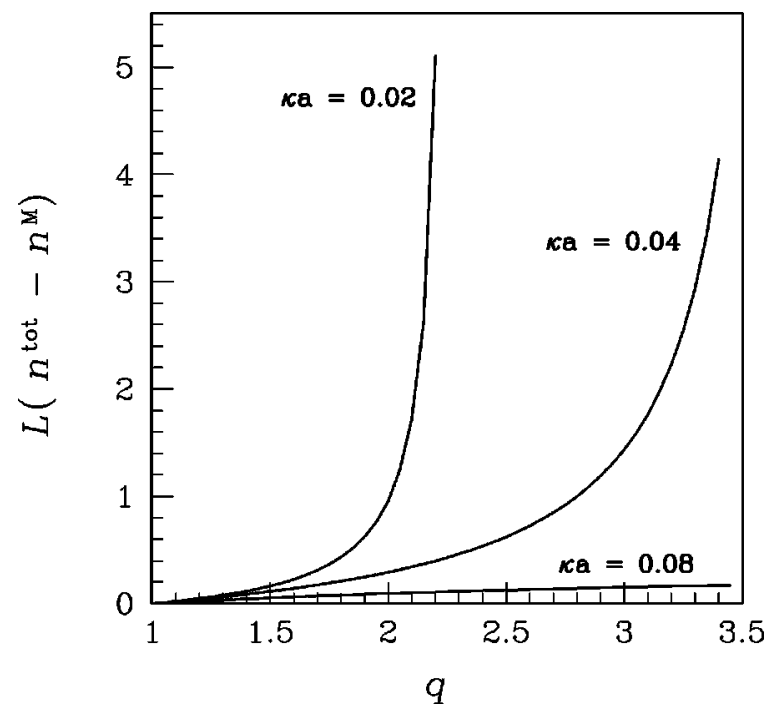

FIG. 2. The excess number of bound counterions $L\left(n_{2}^{\text {tot }}[\rho]\right.$ $-n^{\mathrm{M}}$ ) according to Eq. (4.15), as a function of $q$. The number of excess bound ions is plotted for three salt concentrations corresponding to $\kappa a=0.02,0.04$, and 0.08 . The radius of curvature was calculated according to $l_{e, 2}$, Eq. (4.14), with $z=3, a=4 \AA$, and $l_{0}=500 \AA$.

terions reduces significantly the rigidity of the $\mathrm{PE}$ and usually $l_{e}<0$, indicating an effective attraction between monomers.

The vanishing of the total persistence length $l_{p}$ under certain conditions is alluding to the phenomena of PE collapse. A full consideration of the rod-globule transition requires a more consistent elastic model for the polymer chain than the persistence length prescription used here. Furthermore, Odijk's method for calculating $l_{e}$ does not hold for flexible polymers [28]. However, the condition that total persistence length vanishes, $l_{p}=0$, is the validity limit of the rodlike regime and is indicative of some mechanical instability. For instance, using parameters applicable to DNA chains: $l_{0}=500 \AA$, $3: 1$ salt, $a=1.7 \AA$, and $l_{B}=7 \AA$ we get a DNA collapse at $\kappa^{-1} \simeq 30 \AA$, corresponding to a $3: 1$ salt concentration of about $17 \mathrm{mM}$.

Expanding the density of the bound ions, Eq. (4.7), for small deformations of the chain yields

$$
n_{2}^{\mathrm{tot}}[\rho]=n^{M}\left(1-\frac{1}{8 \kappa^{2} \ln \kappa a} \frac{1}{\rho^{2}}\right)
$$

For a straight chain, $\rho \rightarrow \infty$ and the Manning value $n^{M}$ is obtained. However, more counterions are condensed on a bent polymer with a finite radius of curvature. This enhanced condensation drives a further reduction of the persistence length. For a rodlike polymer, $\rho^{2}=L l_{p} / 3$ and the correction to $n^{M}$ is small and of order $1 / N$. Figure 2 depicts the excess number of bound counterions as a function of $q$ for three salt concentrations (corresponding to $\kappa a=0.02,0.04$, and 0.08).

This difference does, however, have a significant effect on the electrostatic persistence length, because $H_{\mathrm{eff}, 2}$ is a triple integral over the monomers. In order to examine the effect of 
increased condensation, we look at the asymptotic form of Eq. (4.14) for $q=1+\Delta q, \Delta q \ll 1$, in two cases. In the first we allow the density of the bound counterions to be adjusted according to the equilibrium condition with the bulk (this is an expansion of Eq. (4.14) in power of $\Delta q$ ). This is consistent with the general considerations of our paper. In the second case we add a constraint that fixes the density to be according to the Manning theory for all conformations of the polymer. This more restrictive constraint is added in order to make comparisons with other models. Expanding in $\Delta q$ we recalculate $l_{e}$ for both cases

$$
\begin{gathered}
l_{e}=l_{\mathrm{OSF}}\left[1+\mathrm{O}\left(\Delta q^{2}\right)\right], \\
l_{e}^{\mathrm{fixed}}=l_{\mathrm{OSF}}\left[1-[1 / \ln (\kappa a)] \Delta q+\mathrm{O}\left(\Delta q^{2}\right)\right] .
\end{gathered}
$$

The lack of a linear term in $\Delta q$ in the first expression of Eq. (4.16) indicates that corrections to Manning condensation for bent polymer chains have a substantial influence on the persistence length.

An interesting effect is charge inversion, where the total charge of the polymer with the bound counterions changes sign $\left(z n^{\text {tot }}<1 / a\right)$. The persistence length at which charge inversion occurs is given by

$$
l_{p}^{\text {inv }}=\frac{3 z n^{M}}{8 \kappa^{2}\left(a z n^{M}-1\right) \ln \kappa a} \frac{1}{N} .
$$

According to our model, charge inversion will not occur on a long $(N \gg 1)$, rodlike polymer, because $l_{p}^{\text {inv }} \propto 1 / N$.

\section{Higher-order corrections}

In order to examine the convergence of the loop expansion used above, we have calculated the next two orders of approximation: $\left(\psi^{b}\right)^{3}$ and $\left(\psi^{b}\right)^{4}$. The effective interactions are obtained by taking into account terms of higher orders of $\psi^{b}$ in Eq. (4.3). The third and fourth-order terms are given as

$$
\begin{gathered}
H_{3}=-a n_{0} \int d^{3} \mathbf{r} \phi^{m}(\mathbf{r}) \frac{i^{3}}{6}\left\langle\left(\psi^{b}(\mathbf{r})\right)^{3}\right\rangle_{1}, \\
H_{4}=-a n_{0} \int d^{3} \mathbf{r} \phi^{m}(\mathbf{r}) \frac{i^{4}}{4 !}\left\langle\left(\psi^{b}(\mathbf{r})\right)^{4}\right\rangle_{1} \\
-\frac{1}{2} a^{2}\left(n_{0}\right)^{2}\left\{\left\langle\int d^{3} \mathbf{r} \phi^{m}(\mathbf{r}) \frac{i^{2}}{2}\left(\psi^{b}(\mathbf{r})\right)^{2}\right]^{2}\right\rangle_{1} \\
\left.-\left\langle\int d^{3} \mathbf{r} \phi^{m}(\mathbf{r}) \frac{i^{2}}{2}\left(\psi^{b}(\mathbf{r})\right)^{2}\right\rangle_{1}^{2}\right\} .
\end{gathered}
$$

Higher orders of $\psi^{b}$ can be taken into account following the same prescription used above for calculating the chemical potential and the persistence length for the second-order correction. The expression for $l_{e, 3}$ and $l_{e, 4}$ are not detailed here because they are quite complex. However, they do not change the polymer behavior in any qualitative fashion. We find that Eq. (4.14), valid to second order, accounts for most of the deviation from the OSF result. Third- and fourth-order terms represent only a relatively small correction to the second-order one. For instance, for $q>10$ and $\kappa a<0.01$, the third-order correction is less than $4 \%$ of the second-order one. The fourth-order correction is again less than $4 \%$ of the third-order one.

Furthermore, we find that the convergence is better for large $q$ and small $\kappa a$ [45]. This is the more interesting $q$ regime since, for instance, in DNA solutions with trivalent counterions we get roughly $q \simeq 12$.

\section{COMPARISON WITH OTHER MODELS}

Our model is closely related to several previous ones. Ha and Thirumalai have used a similar loop expansion method for calculating the persistence length of polyampholytes [26] and of bundles of polymers [25]. For the case of a single PE, Golestanian et al. [35] took into account thermal fluctuations of the bound counterions density. This is strictly an allfluctuations model, which is expected to become accurate at high temperatures $(q=1+\Delta q, \Delta q \ll 1)$. Their expression for the electrostatic persistence length is [35]

$$
l_{e}^{\text {fluct }}=\frac{l_{B}}{4 q^{2} \kappa^{2} a^{2}[1-(q-1) \ln (\kappa a)]^{2}},
$$

where we have explicitly omitted terms with a higher-order dependence on $\kappa a$.

A second model, suggested by Nguyen et al. [34] assumes that condensed ions are arranged in a Wigner crystal, or a strongly correlated liquid on an infinite cylinder with diameter $d$. This picture becomes accurate at low temperatures $(q \gg 1)$. For the case of no added salt, their expression for the electrostatic persistence length is

$$
l_{e}^{\mathrm{corr}}=l_{B} \sqrt{z}(d / a)^{3 / 2} .
$$

As Nguyen et al. did not take into account the effect of salt (they considered only counterions), we have slightly modified their derivation for the purpose of comparison with our model. Equation (13) of Ref. [34] estimates the interaction energy of an ion with its Wigner-Seitz cell of background charges. Up to a constant of order unity, it is found to be

$$
\epsilon(n) \simeq-\frac{n^{1 / 2} z^{2} e^{2}}{\varepsilon},
$$

where $n$ is the average surface charge density on the cylinder. The only change we include in the above equation is to assume that in the presence of salt, the interaction energy should be proportional to the number of Wigner-Seitz cells that reside within a circle with a radius equal, roughly, to the screening length $\kappa^{-1}$. Up to a constant prefactor, this modification gives

$$
\tilde{\epsilon}(n) \simeq-\frac{n^{1 / 2} z^{2} e^{2}}{\varepsilon} \frac{n}{\kappa^{2}} .
$$

Repeating the derivations of Ref. [34] with this modification of their Eq. (13), and assuming that $d \simeq a$ yields 


$$
l_{e}^{\mathrm{corr}} \simeq-l_{\mathrm{OSF}} \frac{q^{2}}{\sqrt{z}} .
$$

Our loop expansion method can be shown to account qualitatively for both limits of the parameter $q$. As discussed in the preceding section, we find $q$ to be the relevant, temperature dependent parameter that determines the system behavior. This is the reason we expand the results in the two limits $q \gg 1$ and $q \geqslant 1$ rather than low or high temperatures $(\kappa \rightarrow \infty$ or 0$)$. The limits of very low or high temperatures are beyond the validity range of our model which explicitly assumes that $L^{-1} \ll \kappa \ll a^{-1}$. However, experimental systems usually have a large value of $q$ with a finite screening length. For instance, typical parameter values in experiments with DNA segments at room temperature are $l_{B}=7 \AA, a$ $=1.7 \AA, \kappa^{-1}=10-100 \AA$ and $N \geqslant 150$. With trivalent counterions $(z=3)$ we get $q \simeq 12 \gg 1$ and $\kappa L>1$. This is an example where we can consider the relatively high $q$ limit at room temperature.

In order to compare the three models, we expand the expressions for the electrostatic persistence length: ours $l_{e}^{\text {loop }}$; $l_{e}^{\text {fluct }}$ of Ref. [35]; and $l_{e}^{\text {corr }}$ of Ref. [34], in these two limits.

Including terms up to linear order in $\Delta q$ close to $q=1$ we get

$$
\begin{gathered}
l_{e}^{\text {fluct }}=l_{\mathrm{OSF}}\left[1-2 \ln (\kappa a) \Delta q+\mathrm{O}\left(\Delta q^{2}\right)\right], \\
l_{e}^{\text {corr }} \simeq l_{\mathrm{OSF}}\left[-1 / \sqrt{z}-2 / \sqrt{z} \Delta q+\mathrm{O}\left(\Delta q^{2}\right)\right], \\
l_{e}^{\text {loop }}=l_{\mathrm{OSF}}\left[1+\mathrm{O}\left(\Delta q^{2}\right)\right] .
\end{gathered}
$$

However, the calculations of Golestanian et al. assumes that the amount of condensed counterions is according to Manning for all polymer conformations. In the loop calculation, we have relaxed this assumption and took the Manning counterion value only for the completely straight-rod case. For the sake of comparison, we impose now this restriction. This has been done already in Eq. (4.16)

$$
l_{e}^{\text {loop, fixed }}=l_{\mathrm{OSF}}\left[1-\Delta q / \ln (\kappa a)+\mathrm{O}\left(\Delta q^{2}\right)\right] .
$$

With the new restriction, the linear term in $\Delta q$ reappears but with a different coefficient than in $l_{e}^{\text {corr }}$ of Eq. (5.6). The differences in the coefficients, as well as the different dependence on the cutoff distance $a$ is due to the different methods and approximations used in calculating the persistence length.

The second case is that of large $q$, for which the models give

$$
\begin{gathered}
l_{e}^{\text {fluct }}=l_{\mathrm{OSF}}\left[1 /[q \ln (\kappa a)]^{2}+\mathrm{O}\left(1 / q^{3}\right),\right. \\
l_{e}^{\mathrm{corr}}=l_{\mathrm{OSF}}\left[-q^{2} / \sqrt{z}+\mathrm{O}(q)\right], \\
l_{e}^{\mathrm{loop}}=l_{\mathrm{OSF}}\left[-q^{2}+\mathrm{O}(q)\right] .
\end{gathered}
$$

We note that the electrostatic persistence length of the two previous models $[34,35]$ depends very differently on each of the fundamental parameters of the system: the charge density of the polymer $l_{B} / a$, and the valency of the counterions $z$. Furthermore, their expressions do not have similar limits in the two $q$ limits discussed above. However, in the limit $q \gtrsim 1$, our result is similar to that obtained by the fluctuation model [35]. Conversely, in the limit $q \gg 1$, our result resembles that obtained by the correlation model [34]. Some discrepancies are apparent. As explained above, the difference between our model and the fluctuations one is mainly due to the different method used for obtaining the persistence length. The difference with the correlations governed model is mostly due to the discreteness of the charges assumed in Ref. [34] and their specific arrangement in a 2D Wigner lattice.

Our expression for the electrostatic persistence, Eq. (4.14), neither vanishes no diverges in the limits or low or high temperatures. Fluctuation contributions to the electrostatic persistence length vanish in the limit $T \rightarrow 0$. Conversely, correlation contributions vanish in the limit $T \rightarrow \infty$. This illustrates that Eq. (4.14) contains contributions from both fluctuations and correlations.

\section{COMPARISON WITH EXPERIMENTS}

Comparison between our expression for the persistence length, Eq. (4.14), with that obtained in experiments [7-11] is difficult. Although $l_{e, 2}$ correctly predicts that the persistence length should be smaller than OSF, it seems that the reduction we obtain is too large as compared with experiments. Actually, at present, we are not aware of any other theoretical modeling which explains quantitatively the experimental data.

Measurements of rigid PEs usually involve short DNA segments. Experiments show that adding of very small amounts of multivalent counterions greatly reduce the persistence length of DNA, $l_{p}=l_{0}+l_{e}$ even bellow its bare value $l_{0}[7,10,50]$, indicating a negative electrostatic persistence length. However, substituting DNA parameters $(a \simeq 1.7 \AA$, $l_{0} \simeq 500 \AA$ ) and salt concentrations common to experiments (10 $\AA<\kappa^{-1}<100 \AA$ ) into the total persistence length $l_{p}$ of Eq. (4.14), we find that DNA should collapse $\left(l_{p}<0\right)$, for $z=3$ or 4 . This does not agree with experiments where the DNA is still in the rigid-rod limit for the same system parameters.

This discrepancy may be caused by several important experimental features which are neglected in our model as well as in Refs. [34,35]. DNA segments are prepared in a buffer which stabilizes the solution $p \mathrm{H}$ and removes free divalent calcium ions [50]. The buffer itself contributes a finite, nonnegligible concentration of monovalent ions. In a second stage, multivalent ions such as spermidine $(z=3)$ or spermine $(z=4)$ are added. In experiments, the monovalent salt concentration may be much higher than the multivalent one, making the contribution of the multivalent salt to the screening length $\kappa^{-1}$ quite negligible. In the model we did not take into account mixtures of monovalent and multivalent ions.

According to the Manning-Oosawa model which was employed by us, entropy considerations dictate that condensation of multivalent counterions is much more favorable than monovalent ones. At low concentrations of multivalent salts, 
experiments clearly show that this is not always the case [50-52]. At low but finite polymer and multivalent salt concentrations (as is usually the case in experiments), entropy and the finite size of the counterions (spermidine and spermine are relatively large molecules) prohibit multivalent counterions from condensing on the chain. Generalization of the Manning-Oosawa model to account for this effect is not straightforward.

In experiments the change from a rigid rodlike behavior to a flexible one is highly sensitive to the multivalent concentration $[7,10]$. The above discussion emphasizing the deviation from the Manning-Oosawa model may also explain this changeover. At low concentrations of multivalent ions, less counterions are condensed than according to the Manning value. Hence, the chain is still rigid in disagreement with our prediction. At higher multivalent salt and polymer concentrations, where the Manning value for condensation on a cylinder $n^{M}$ holds, the DNA is completely collapsed, making both theory and experimental measurements of persistence length useless. Therefore, the main difficulty in our (and similar) models is the small window of parameter values where the model can be applied. Experiments with DNA do not, in general, fall in this window due to the strong charging of the chains.

We briefly mention other features not considered in our model, and which may influence the persistence length. They include the finite size of the counterions [53], the ordering of the charges along the polymer chain $[54,55]$, the concentration profile of the condensed ions around the polymer [43] and other, more specific, details of the polymer type and ions used in experiments [56].

\section{SUMMARY}

We have found significant corrections to the persistence length of a single, stiff, strongly charged and long PE, as compared to the standard mean-field result of OdijkSkolnick-Fixman. Our method takes into account both correlations between condensed ions and thermal fluctuations. At low salt concentrations, the calculated electrostatic persistence length $l_{e, 2}$ is proportional to $l_{\mathrm{OSF}}$. However, the prefactor, which depends on $q=z l_{B} / a$, drastically changes the system behavior. For $q \leqslant 1, l_{e, 2}=l_{\mathrm{OSF}}$ is obtained exactly. For $1<q<2$, the electrostatic persistence length $l_{e, 2}$ is positive, indicating an effective repulsion between the monomers. For $q>2$, the interaction becomes attractive causing a reduction in the chain stiffness, $l_{e, 2}<0$. This observation is in agreement with the reduction in persistent length observed qualitative in experiments with multivalent counterions and strongly charged polymers.

We compared our result for the electrostatic persistence length, Eq. (4.14), with two previous models and found that our model takes into account both thermal fluctuations and correlations between bound counterions. Our model qualitatively agrees with the fluctuation model in the $q \gtrsim 1$ limit, with the correlation model in the $q \gg 1$ limit, and may offer new insight for intermediate values of $q$.

It is interesting to note that $q=2$ corresponds to the case where the average electrostatic interaction between bound ions equals $k_{B} T$. This means that for $1<q<2$, thermal fluctuations are expected to dominate over correlations. On the other hand, for $q>2$, correlations become more significant. Our conclusion is that although thermal fluctuations reduce the (mean-field) electrostatic repulsion between monomers, they are not sufficient to induce effective attraction. In order to correctly describe the attractive, collapsed case, correlations between counterions have to be included.

We have also obtained the average density of bound ions and found that more counterions condense on the chain than is predicted by the Manning-Oosawa model. The increased condensation has a significant effect on the persistence length and cannot be neglected. Furthermore, we have estimated the conditions under which collapse of a rigid PE may occur. The results are reasonable and relate, at least qualitatively, to the phenomena of DNA condensation. As explained in previous sections, our theory cannot yet be directly compared with experimental results (in particular with DNA).

We believe that additional work is needed to shed more light on the mechanical instability of the chain, indicating a rod-globule transition. Different, more complex, methods are required in order to calculate the persistence length of flexible chains. For instance, the validity range of some variational methods are known to be wider than that of the OSF theory $[27,28]$, and may better apply to flexible chains. Moreover, the entire notion of persistence length for describing the chain elastic properties breaks down close to the instability. The vanishing of the persistence length indicates that the method is no longer consistent, and higher powers of the radius of local curvature $\partial^{2} \mathbf{R} / \partial s^{2}$ have to be taken into account.

DNA experiments are usually performed for polymer and salt concentrations requiring a more detailed examination of the counterion condensation phenomena than the simplified Manning-Oosawa model. Some effects that are unique to mixtures of different types of ions need to be taken into account. A quantitative analysis of this phenomenon requires further and rather detailed considerations and will be presented elsewhere.

\section{ACKNOWLEDGMENTS}

We would like to thank I. Borukhov, H. Diamant, A. Grosberg, B.-Y. Ha, M. Kozlov, R. Mints, R. Netz, T. Odijk, H. Orland, Y. Rabin, B. Shklovskii, D. Thirumalai, T. Witten, and in particular, Y. Burak for useful discussions and correspondence. Partial support from the U.S.-Israel Binational Science Foundation (BSF) under Grant No. 98-00429 and from the Israel Science Foundation founded by the Israel Academy of Sciences and Humanities-centers of Excellence Program, and under Grant No. 210/02 is gratefully acknowledged. 
[1] T.A. Witten and P. Pincus, Europhys. Lett. 3, 315 (1987).

[2] J.-L. Barrat and J.-F. Joanny, Adv. Chem. Phys. 94, 1 (1996).

[3] F. Oosawa, Polyelectrolytes (Marcel Dekker, New York, 1971).

[4] H. Yamakawa, Modern Theory of Polymer Solutions (Harper and Row, New York, 1971).

[5] A.V. Dobrynin, R.H. Colby, and M. Rubinstein, Macromolecules 28, 1859 (1995).

[6] P.G. de Gennes, P. Pincus, R.M. Velasco, and F. Brochard, J. Phys. (Paris) 37, 1461 (1976).

[7] C.G. Baumann, S.B. Smith, V.A. Bloomfield, and C. Bustamante, Proc. Natl. Acad. Sci. U.S.A. 94, 6185 (1997).

[8] W.J. Walczak, D.A. Hoagland, and S.L. Hsu, Macromolecules 29, 7514 (1996).

[9] T. Hugel, M. Grosholz, H. Clausen-Schaumann, A. Pfau, H. Gaub, and M. Seitz, Macromolecules 34, 1039 (2001).

[10] P.J. Hagerman, Biopolymers 20, 1503 (1981).

[11] M.N. Spiteri, F. Boué, A. Lapp, and J.P. Cotton, Phys. Rev. Lett. 77, 5218 (1996).

[12] U. Micka and K. Kremer, Europhys. Lett. 38, 279 (1997).

[13] R.G. Winkler, M. Gold, and P. Reineker, Phys. Rev. Lett. 80, 3731 (1998).

[14] M.O. Khan and B. Jönsson, Biopolymers 49, 121 (1999).

[15] M.J. Stevens and K. Kremer, Phys. Rev. Lett. 71, 2228 (1993).

[16] V. Degiorgio, F. Mantegazza, and R. Piazza, Europhys. Lett. 15, 75 (1991).

[17] M. Schmidt, Macromolecules 24, 5361 (1991).

[18] M. Tricot, Macromolecules 17, 1698 (1984).

[19] N.V. Brilliantov, D.V. Kuznetsov, and R. Klein, Phys. Rev. Lett. 81, 1433 (1998).

[20] F.J. Solis and M. Olvera de la Cruz, J. Chem. Phys. 112, 2030 (2000).

[21] P.L. Hansen, D. Svensek, V.A. Parsegian, and R. Podgornik, Phys. Rev. E 60, 1956 (1999).

[22] V.A. Bloomfield, Curr. Opin. Struct. Biol. 6, 334 (1996), and references therein.

[23] B.-Y. Ha and D. Thirumalai, J. Chem. Phys. 110, 7533 (1999).

[24] B.-Y. Ha and D. Thirumalai, Macromolecules 28, 577 (1995).

[25] B.-Y. Ha and D. Thirumalai, e-print cond-mat/0208466.

[26] B.-Y. Ha and D. Thirumalai, J. Phys. II (France) 7, 877 (1997).

[27] R.R. Netz and H. Orland, Eur. Phys. J. B 8, 81 (1999).

[28] J.-L. Barrat and J.-F. Joanny, Europhys. Lett. 24, 333 (1993).

[29] H. Li and T.A. Witten, Macromolecules 28, 5921 (1995).

[30] T. Odijk, J. Polym. Sci. Part B: Polym. Phys. 15, 477 (1977).

[31] J. Skolnick and M. Fixman, Macromolecules 10, 944 (1977).

[32] M. Fixman, J. Chem. Phys. 76, 6346 (1982).
[33] M. Le Bret, J. Chem. Phys. 76, 6243 (1982).

[34] T.T. Nguyen, I. Rouzina, and B.I. Shklovskii, Phys. Rev. E 60, 7032 (1999).

[35] R. Golestanian, M. Kardar, and T.B. Liverpool, Phys. Rev. Lett. 82, 4456 (1999).

[36] G.S. Manning, J. Chem. Phys. 51, 954 (1969).

[37] A. Diehl, H.A. Carmona, and Y. Levin, Phys. Rev. E 64, 011804 (2001)

[38] A.W.C. Lau, D. Levine, and P. Pincus, Phys. Rev. Lett. 84, 4116 (2000).

[39] M. Kardar and R. Golestanian, Rev. Mod. Phys. 71, 1233 (1999).

[40] Y. Levin, J.J. Arenzon, and J.F. Stilck, Phys. Rev. Lett. 83, 2680 (1999).

[41] T.T. Nguyen, A.Y. Grosberg, and B.I. Shklovskii, in Electrostatic Effects in Soft Matter and Biophysics, Vol. 46 of NATO Science Series II: Mathematics, Physics and Chemistry, edited by C. Holm, P. Kekicheff, and R. Podgornik (Kluwer Academic, Dordrecht, 2001), p. 469.

[42] G. Ariel and D. Andelman, Europhys. Lett. (to be published).

[43] M. Le Bret and H. Zimm, Biopolymers 23, 287 (1984).

[44] R.R. Netz and H. Orland, Europhys. Lett. 45, 726 (1999).

[45] R.R. Netz, Euro. Phys. J 5, 557 (2001).

[46] I. Borukhov, D. Andelman, and H. Orland, Eur. Phys. J. B 5, 869 (1998).

[47] H. Diamant and D. Andelman, Macromolecules 33, 8050 (2000).

[48] Since the Manning condensation model is strictly a mean-field approximation, it cannot be valid close to the condensation value $q=1$, where fluctuations dominate.

[49] Taking into account higher orders of $\psi^{b}$ is not expected to change the Manning result for a single cylinder. See, e.g., B.-Y. Ha and A.J. Liu, Phys. Rev. Lett. 79, 1289 (1997).

[50] E. Raspaud, M. Olvera de la Cruz, and F. Livolant, Biophys. J. 74, 381 (1998).

[51] J. Pelta, F. Livolant, and J.-L. Sikorav, J. Biol. Chem. 10, 5656 (1996).

[52] M. Saminathan, T. Antony, A. Shirahata, L.H. Sigal, T. Thomas, and T.J. Thomas, Biochemistry 38, 3821 (1999).

[53] H.-A. Tajmir-Riahi, M. Naoui, and R. Ahmad, Biopolymers 33, 1819 (1993).

[54] A.A. Kornyshev and S. Leikin, Phys. Rev. Lett. 84, 2537 (2000).

[55] W.H. Braunlin and Q. Xu, Biopolymers 32, 1703 (1992).

[56] V.A. Bloomfield, Biopolymers 31, 1471 (1991). 